\title{
WHITE DWARF AND NEUTRON STAR INTERIOR SOLUTIONS IN THE NONSYMMETRIC GRAVITATIONAL THEORY
}

\author{
L. M. CAmpbell, ${ }^{1}$ J. W. Moffat, ${ }^{2}$ and P. Savaria ${ }^{2}$ \\ Received 1990 June 6; accepted 1990 October 11
}

\begin{abstract}
Spherically symmetric, static, numerical solutions of the field equations of the nonsymmetric gravitational theory (NGT) for dense, homogeneous, cold matter with a stiff equation of state are presented in graphic form. As in Einstein's general relativity theory (GRT), two types of stable objects are predicted, corresponding to white dwarfs and neutron stars. The NGT makes these less stable as it effectively softens the equation of state, decreasing their mass and radius. The requirement of stability sets upper limits on the strength of the coupling of ordinary matter (protons, neutrons, and electrons) to NGT forces, and on the total NGT charge of such stars, which rule out that kind of matter as a source of NGT effects strong enough to account for the discrepancies between observed periastron shifts in some main-sequence binary systems and the predictions of Einstein's theory.
\end{abstract}

Subject headings: gravitation - stars: interiors - stars: neutron — stars: white dwarfs

\section{INTRODUCTION}

The exterior spherically symmetric solution of the nonsymmetric gravitational theory (NGT) (Moffat 1979; for a review, see Moffat 1991) differs markedly from that derived in Einstein's general relativity theory (GRT). In the NGT, a spherically symmetric source acts on the surrounding spacetime geometry not only through its mass, but also through a new quantity whose value, just like the mass, must be adjusted to fit observational data. This quantity, which arises as a constant of integration, has dimensions of length squared and, for this reason, is denoted $L^{2}$. Test bodies move (Moffat 1987) in the field of the source under the influence of a repulsive force resulting from the background geometry - thus depending only on the source - as well as a tensor force that couples the test body to the source. Both forces decrease with the fifth power of the distance to the source.

When the equations of motion are solved, the NGT is found (Moffat 1987; Moffat \& Woolgar 1988) to predict an apsidal motion of orbits slower than that expected in the GRT. The magnitude of the NGT effect is a function of the difference between the $L^{2}$-tomass ratios of the bodies, and it disappears when those ratios are equal. In this way, the nonsymmetric theory may account (Moffat 1989) for the discrepancies reported (see the references in Moffat 1989) in several close binary systems between the observed periastron shift and the predictions of the GRT. Two such systems, DI Her and AS Cam, deviate from the GRT by more than 20 standard deviations (Guinan \& Maloney 1985; Guinan et al. 1987; Maloney et al. 1989). Another well-observed periastron shift, that of the binary pulsar PSR 1913 + 16 (Weisberg \& Taylor 1984; Taylor \& Weisberg 1989), does agree closely with the GRT, constraining (Krishner 1985; Moffat 1987) the value of $L^{2}$ for neutron stars, but there now appears (Damour $\&$ Taylor 1990) to be a possible disagreement between the gravitational radiation emitted by this system and the level predicted by the GRT. Here again, there exist (Moffat 1990a) values of $L^{2}$ and of the masses for both objects that can resolve this difference and still yield an excellent fit to the periastron shift.

The question then arises whether NGT admits interior solutions consistent with the exterior solutions that account for these observations. One of us has shown (Savaria 1989) that in main-sequence stars composed of a homogeneous mixture of baryons and leptons as NGT sources, the extra NGT structure needed to meet all the observational constraints on their $L^{2}$ values has essentially no influence on their pressure and density, and therefore on their mass, radius, and luminosity. Results of Earth-based "fifth force" experiments, however, seem to rule out normal matter as an effective NGT source capable of explaining the anomalous behavior of main-sequence binaries: the size of the coupling of matter to NGT fields that they allow is too small by some three orders of magnitude. A model with weakly interacting massive particles as NGT sources, proposed by Moffat (1989) to obviate this difficulty, is currently under investigation to find out whether there exist acceptable interior solutions compatible with it.

This paper focuses on white dwarf and neutron star interiors in the NGT. Working under the assumption of homogeneous normal matter composition, we obtain numerical solutions to the set of equations (Savaria 1989) for the spherically symmetric interior problem as applied to those cases. Section 2 recalls briefly the findings of Savaria (1989). Sections 3, 4, and 5 discuss the equation of state, the stability criteria, and the numerical integration procedure. Sections 6 and 7 present solutions pertaining to white dwarfs and neutron stars, respectively. These are displayed as graphs so as to illustrate most clearly how the NGT differs from the GRT. Section 8 concludes with a summary of our main results and the constraints they put on the strength of the coupling of matter to the NGT.

\section{THEORETICAL FRAMEWORK}

The reader should refer to Savaria (1989) for a fuller account of the general formalism of the NGT as applied to this problem. The field equations are derived by varying the Lagrangian density

$$
\mathscr{L}=\mathbf{g}^{\mu \nu} R_{\mu \nu}(W)-\frac{8 \pi G}{c^{4}} g^{\mu \nu} \mathbf{T}_{\mu \nu}+(8 \pi / 3) W_{\mu} \mathbf{S}^{\mu},
$$

\footnotetext{
${ }^{1}$ Instituto de Optica CSIC, 28006 Madrid, Spain.

${ }^{2}$ Department of Physics, University of Toronto, Toronto, Canada M5S 1A7.
} 
where $\mathbf{g}^{\mu \nu}$ is the nonsymmetric contravariant fundamental tensor density and $W$ is an a priori unconstrained nonsymmetric connection with torsion vector $W_{\mu}$. Tensor densities are represented by boldface type.

The tensor $R_{\mu \nu}(W)$ is defined by

$$
R_{\mu \nu}(W)=W_{\mu \nu, \rho}^{\rho}-\frac{1}{2}\left(W_{\mu \rho, v}^{\rho}+W_{v \rho, \mu}^{\rho}\right)+W_{\mu \nu}^{\rho} W_{\rho \sigma}^{\sigma}-W_{\mu \sigma}^{\rho} W_{\rho \nu}^{\sigma} .
$$

Instead of $W$, one normally uses a connection $\Gamma$ such that $\Gamma_{\mu \nu}^{\lambda} \equiv W_{\mu \nu}^{\lambda}+\frac{2}{3} \delta_{\mu}^{\lambda} W_{v}$. Then the field equations which follow from varying $\mathscr{L}$ are

$$
\begin{gathered}
G_{\mu \nu}(\Gamma) \equiv R_{\mu \nu}(\Gamma)-\frac{1}{2} g_{\mu \nu} g^{\alpha \beta} R_{\alpha \beta}(\Gamma)=\frac{8 \pi G}{c^{4}} T_{\mu \nu}-\frac{2}{3} W_{[\mu, v]}+\frac{1}{3} g_{\mu \nu} g^{\alpha \beta} W_{[\alpha, \beta]} \\
\mathbf{g}^{\mu v}{ }_{, \lambda}+\mathbf{g}^{\rho v} \Gamma_{\rho \lambda}^{\mu}+\mathbf{g}^{\mu \rho} \Gamma_{\lambda \rho}^{v}-\mathbf{g}^{\mu v} \Gamma_{(\lambda \rho)}^{\rho}+(4 \pi / 3)\left(\mathbf{S}^{\nu} \delta_{\lambda}^{\mu}-\mathbf{S}^{\mu} \delta_{\lambda}^{v}\right)=0 \\
\mathbf{g}^{[\mu v]}{ }_{, v}=4 \pi \mathbf{S}^{\mu} .
\end{gathered}
$$

Parentheses and square brackets enclosing indices stand for symmetrization and antisymmetrization, respectively.

These field equations, together with the Bianchi identities of the theory, lead to the following matter-response equations:

$$
\frac{1}{2}\left(g_{\rho \mu} \mathbf{T}_{, v}^{v \mu}+g_{\mu \rho} \mathbf{T}_{, v}^{\mu v}\right)+\mathbf{T}^{\mu v}[\mu \nu, \rho]+\frac{1}{3} W_{[\rho, v]} \mathbf{S}^{v}=0 . \quad[\mu v, \rho] \equiv \frac{1}{2}\left(g_{\mu \rho, v}+g_{\rho v, \mu}-g_{\mu v, \rho}\right) .
$$

Matter is assumed to consist of a perfect fluid of homogeneous chemical composition, matter-density $\rho$ and pressure $p$, in which all particles move with a common four-velocity $u^{\mu}$. The energy-momentum tensor appropriate to this fluid takes the same form as in the GRT (Vincent 1985; Campbell 1988):

$$
T^{\mu v}=(\rho+p) u^{\mu} u^{v}-p g^{\mu v} .
$$

Matter couples (see eq. [1]) to the NGT partly through a conserved current density $\mathbf{S}^{\mu}$. Macroscopically, it is useful to think of $S^{\mu}$ as composed of particles, each with their own NGT coupling strengths. Thus,

$$
S^{\mu}=\sum_{i} f_{i}^{2} n_{i} u^{\mu}
$$

$f_{i}^{2}$ being the coupling strength and $n_{i}$ the rest-number density of the $i$ th species of particle coupled to $W_{\mu}$. We can rewrite this current so that

where

$$
S^{\mu}=\frac{f_{\mathrm{eff}}^{2}}{m_{n}} \rho_{0} u^{\mu}
$$

$$
f_{\text {eff }}^{2}=\frac{\sum_{i} f_{i}^{2} n_{i}}{\sum_{i}\left(m_{i} / m_{n}\right) n_{i}},
$$

with $\rho_{0}$ the rest-mass density and $m_{n}$ the mass of the nucleon. Because of the static character of our solutions, $u^{0}=\left(g_{00}\right)^{-1 / 2}$, and $u^{i}=0(i=1,2,3)$.

In a static spherically symmetric geometry, the line element takes the form

$$
d s^{2}=\gamma(r) c^{2} d t^{2}-\alpha(r) d r^{2}-r^{2} d \Omega^{2} .
$$

Equation (3) then leads to a system of three first-order differential equations (Savaria 1989, 1991). With the prime denoting differentiation with respect to $r$,

$$
\begin{aligned}
\left(l^{2}\right)^{\prime} & =4 \pi r^{2}(1+s)\left[\left(1+l^{4} / r^{4}\right)\left(1-2 G m / c^{2} r\right)\right]^{-1 / 2} f_{\text {eff }}^{2} \rho_{0} / m_{n}, \\
m^{\prime} & =4 \pi \rho(1+s) r^{2}-m s / r^{3} \\
r p^{\prime} & =-\left(\rho c^{2}+p\right)(1+s)\left[\left(1-\frac{2 G m}{c^{2} r}\right)^{-1}\left(\frac{4 \pi G p r^{2}}{c^{4}}+\frac{G m}{c^{2} r}\right)-2 \frac{l^{4}}{r^{4}}\left(1+\frac{l^{4}}{r^{4}}\right)^{-1}\right]-2 s p-s m c^{2} / \pi r^{3} .
\end{aligned}
$$

The unknown $l^{2}$ is simply the total NGT charge enclosed within a spherical volume of radius $r, s=d l^{4} / d r^{4}$, and $\alpha$ is given in terms of $m$ and $s$ by $\alpha=(1+s)^{2} /\left(1-2 G m / c^{2} r\right)$. The function $m(r)$ measures (Savaria 1991) the total energy contained within a sphere of radius $r$. Another differential equation that determines $\gamma(r)$ has been omitted here, since we do not need it to obtain the mass, radius, and NGT charge of a body. The reader should refer to Savaria $(1989,1991)$ for a fuller discussion of equations $(10)$, our aim in this paper being to present numerical solutions of this system.

\section{EQUATION OF STATE}

In order to obtain solutions for equations (10), it is necessary to specify an equation of state (EOS), $p=p(\rho)$, appropriate to the type of matter under consideration, here cold matter. At densities below $2 \times 10^{10} \mathrm{~g} \mathrm{~cm}^{-3}$, we shall use an EOS (Chandrasekhar 1939) describing a fluid composed of protons, neutrons, and electrons, treated as noninteracting degenerate Fermi gases. Electrostatic contributions, which are important only at densities below $10^{4} \mathrm{~g} \mathrm{~cm}^{-3}$, have been ignored at the cost of some inaccuracy in the radius of white dwarfs. This has very little effect on the mass and NGT charge of the stars with higher central density that are of interest here. 
Above $2 \times 10^{10} \mathrm{~g} \mathrm{~cm}^{-3}$ and up to $10^{16} \mathrm{~g} \mathrm{~cm}^{-3}$, we work with a variant (Pandharipande, Pines, \& Smith 1976) of the mean field EOS that yields the highest mass $\left(2.7 M_{\odot}\right)$ for neutron stars in the GRT. Repulsive core interactions may not be as large as in that model, and effects that soften the EOS underestimated, so that the maximum GRT mass may not exceed $\approx 2 M_{\odot}$. But, as we shall see, the NGT maximum mass decreases with the coupling $f_{\text {eff }}^{2}$, so that using this EOS will allow us to find an upper bound on $f_{\text {eff }}^{2}$ that is independent of the equation of state.

Convenient as it is, the Chandrasekhar EOS can only be an approximation in the outer layers $\left(\rho<10^{10} \mathrm{~g} \mathrm{~cm}^{-3}\right)$ of bodies whose central density reaches $10^{14}-10^{15} \mathrm{~g} \mathrm{~cm}^{-3}$. Nevertheless, it is quite adequate for our purposes since these regions contribute so little to the total mass and NGT charge. Indeed, our GRT solutions for neutron stars, which use the Chandrasekhar EOS in the low-density layers, show no difference in mass and radius from those found (see, for example, Baym, Bethe, \& Pethick 1971a and Baym, Pethick, \& Sutherland 1971b) with the help of more accurate EOS in this regime.

Figure 1 shows the EOS used in all our computations, over densities ranging from $10^{5}$ to $10^{16} \mathrm{~g} \mathrm{~cm}^{-3}$.

\section{STABILITY CONDITIONS}

We shall implement the same stability criteria as in Newton and Einstein gravitational theories. This involves the standard method (Harrison et al. 1965) of looking at the effect of a periodic perturbation, $\xi(r, t)=\sum_{n} \xi_{n}(r) e^{i \omega_{n} t}$, on a solution. The frequencies $\omega_{n}$ of the resulting oscillation must all be real for the solution to be stable. For each value of $f_{\text {eff }}^{2}$, equilibrium solutions can be parameterized by a value of the density, $\rho_{c}$, at $r=0$. Critical points between stability and instability always occur at extrema of the mass versus density curve, where $\partial M / \partial \rho_{c}=0$. A necessary-but not sufficient-condition for stability is that $\partial M / \partial \rho_{c}>0$.

At each extremum of $M\left(\rho_{c}\right)$, one $\omega_{n}^{2}$ or another must change sign. To find out which, one examines the dependence of the radius $R$ of the solution on $\rho_{c}$. If $\partial R / \partial \rho_{c}>0$ where $\partial M / \partial \rho_{c}=0, n$ is odd; otherwise it is even. Since modes with $\omega_{n}^{2}<0$ are unstable while those with $\omega_{n}^{2}>0$ are stable, and since also $\omega_{n}^{2}<\omega_{n+1}^{2}$ for all $n$, it follows that the $\omega_{n}^{2}$ must change sign in sequence, and that solutions are stable if and only if $\omega_{0}^{2}>0$. Given one known stable equilibrium solution, it is then possible to determine whether any other solution is stable. Here, as in the GRT, solutions at low central densities $\left(\rho_{c} \approx 10^{6} \mathrm{~g} \mathrm{~cm}^{-3}\right)$ are assumed stable. This leads, in the NGT as well as in the GRT, to the prediction of the existence, at higher $\rho_{c}$, of two groups of stable bodies, white dwarf stars and neutron stars.

Although we have not been able to show explicitly that harmonic perturbations of the NGT system (10) obey a Sturm-Liouville equation, we feel confident that the above stability conditions still apply in the NGT, and this for the following reason. As will be evident from the graphs of the solutions, small variations of $f_{\text {eff }}^{2}$ induce only small changes in the mass, the radius, and the total NGT charge $L^{2}$, anywhere in the stable solution space as determined from those criteria. This is also the case for changes in $\rho_{c}$ at every value of $f_{\text {eff }}^{2}$ giving rise to stable solutions. As $f_{\text {eff }}^{2}$ is increased from its GRT value of zero, stable NGT solutions deviate smoothly from the GRT solutions.

\section{OUTLINE OF THE NUMERICAL INTEGRATION PROCEDURE}

Integration of system (10) was performed on a computer with the help of a sophisticated numerical solver for systems of first-order differential equations called LSODE (Hindmarsh 1980). Two pieces of data were specified at $r=0$ : the matter density $\rho_{c}$ and the

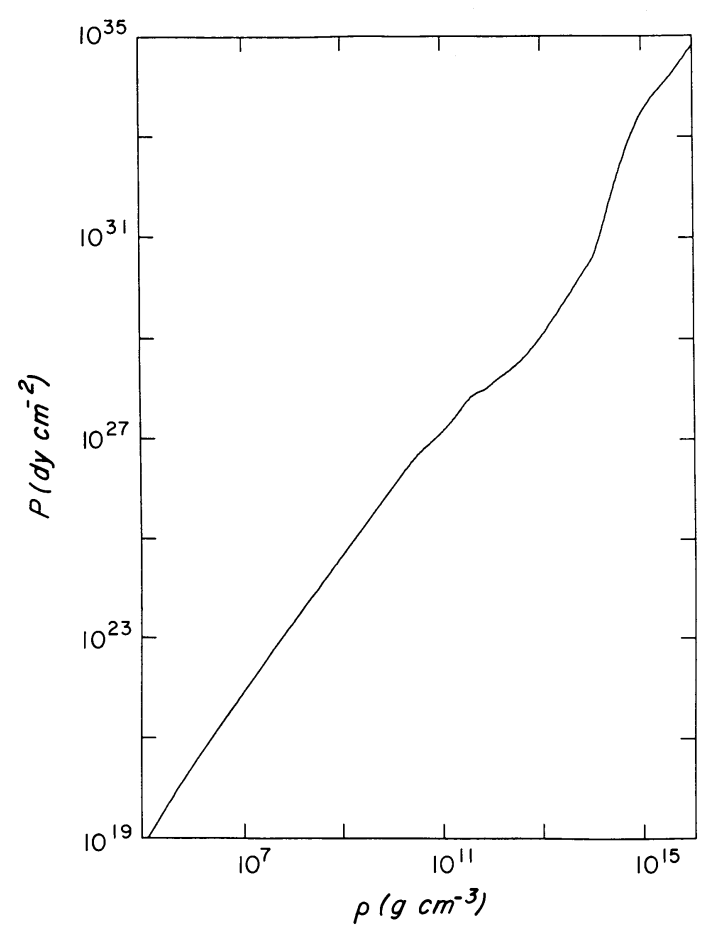

Fig. 1.-The equation of state for cold matter obtained by joining the Chandrasekhar equation of state, for $\rho<10^{11} \mathrm{~g} \mathrm{~cm}^{-3}$, to the higher density mean-field equation of state. 
effective NGT coupling strength $f_{\text {eff }}^{2}$. From these, the mass $M$, radius $R$, and NGT charge $L^{2}$ of the body could be uniquely determined. As system (10) diverges formally at the center, an analytic solution obtained (Savaria 1989) by expanding it around $r=0$, allowed us to start the integration a small distance away from the center. This solution is reproduced here for completeness. If $l_{0} \equiv 4 \pi f_{\text {eff }}^{2}\left(\rho_{0}\right)_{c} / 3 m_{n}$, then

$$
\begin{aligned}
l^{2}(r) & =l_{0} r^{3}+O\left(r^{4}\right)+\cdots \\
s(r) & =\frac{3}{2} l_{0}^{2} r^{2}+O\left(r^{4}\right)+\cdots \\
m(r) & =\frac{4 \pi}{3} \rho_{c} r^{3}+O\left(r^{5}\right)+\cdots \\
p(r) & =p_{c}-\left[2 \pi \frac{G}{c^{4}}\left(\rho_{c} c^{2}+p_{c}\right)\left(\frac{1}{3} \rho_{c} c^{2}+p_{c}\right)+\frac{1}{2} p_{c} l_{0}^{2}\right] r^{2}+O\left(r^{4}\right)+\cdots
\end{aligned}
$$

which yields $\alpha(r) \approx 1+\left(8 \pi G \rho_{c} / 3 c^{2}+3 l_{0}^{2}\right) r^{2}+O\left(r^{4}\right)$.

The integration can then proceed forward up to the surface, defined as the point $r=R$ where the matter parameters, $\rho, p$, and $S^{0}$ (or, equivalently, $s$ ) all vanish.

The total mass $M$ of the star is determined from the matching condition on $\alpha_{\text {in }}$ and $\alpha_{\text {ext }}\left(\right.$ Moffat 1979) at the surface: $\alpha_{\text {in }}(R)=$ $\alpha_{\text {ext }}(R)=(1-2 M / R)^{-1}$.

The mass could also be calculated by direct volume integration (Savaria 1989):

$$
M=\left(1+\frac{L^{4}}{R^{4}}\right)^{-1} \int_{\mathrm{star}} \sqrt{-g}\left\{\rho+\frac{3 p}{c^{2}}+\left(\frac{l^{4}}{r^{4}}\right)\left[\frac{m}{\pi r^{3}}+\left(3 \rho+\frac{p}{c^{2}}\right)\right]\right\} d r d \theta d \phi,
$$

where $\sqrt{-g}=\left[(\alpha \gamma) /\left(1+l^{4} / r^{4}\right)\right]^{1 / 2} r^{2} \sin \theta$. Equation (12), however, has the disadvantage of requiring knowledge of $g_{00}$ [i.e., $\left.\gamma(r)\right]$, which entails solving an extra differential equation. This was indeed done at the beginning, but once it was established that the masses obtained from the two methods always agreed, we used exclusively the boundary condition.

The total NGT charge $L^{2}$ was identified with the value of the parameter $l^{2}$ (eq. [10a]) at $r=R$.

\section{WHITE DWARF SOLUTIONS}

The effect of the NGT is perhaps best illustrated by a graph of the mass $M$ versus central density $\rho_{c}$. Figure 2 shows this for a range of $\rho_{c}$ corresponding to stable white dwarf stars. The GRT solution has its maximum mass $M_{\max }$ of $1.42 M_{\odot}$ at $\rho_{c}=2.7 \times 10^{10}$ $\mathrm{g} \mathrm{cm}^{-3}$. The three NGT curves result from choosing three representative values for $f_{\text {eff }}^{2}$. They all coincide with the GRT curve at low $\rho_{c}$ but then start to deviate from it so that, shortly thereafter, they reach their own maximum at a value of $M$ which decreases as $f_{\text {eff }}^{2}$ increases. Beyond that maximum the solutions are unstable: $\partial M / \partial \rho_{c}<0$ and the frequency of the fundamental perturbed mode has become imaginary. The slope becomes positive again at higher $\rho_{c}$, but $\partial R / \partial \rho_{c}>0$ at that minimum, making the second mode unstable.

We observe that the NGT reduces the upper limits on both the mass and the central density of white dwarf stars. The latter especially depend rather strongly on $f_{\text {eff }}^{2}$. Between $f_{\text {eff }}^{2}=0(\mathrm{GRT})$ and $f_{\text {eff }}^{2}=6 \times 10^{-42} \mathrm{~cm}^{2}, M_{\max }$ has decreased from $1.42 M_{\odot}$ to

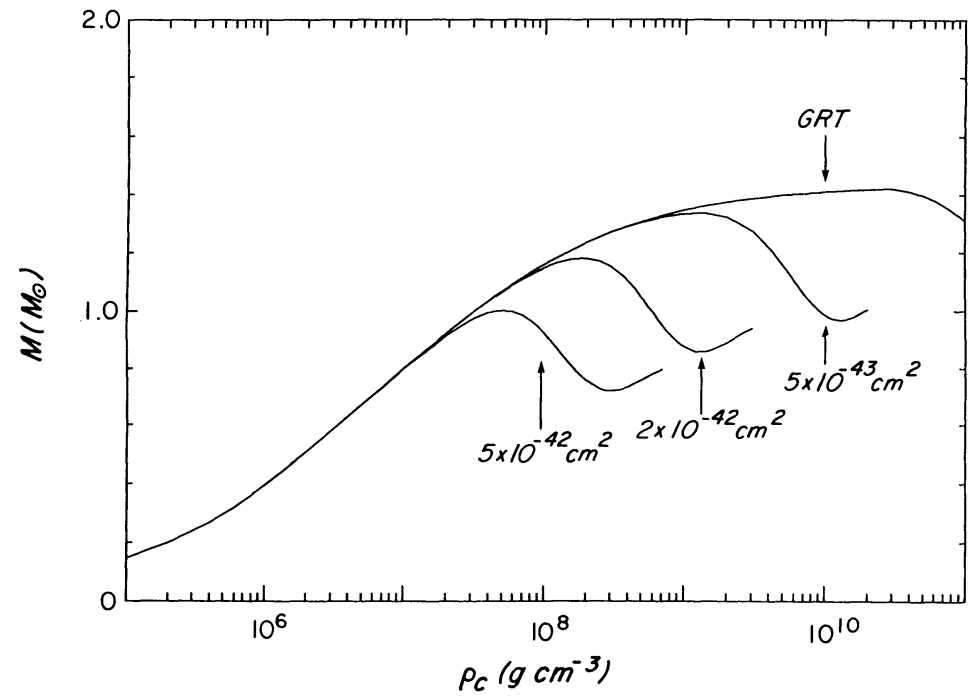

FIG. 2.--The variation of the mass of white dwarfs with central density in the GRT and in the NGT with three values of $f_{\text {eff }}^{2}$ 
$1 M_{\odot}$, but $\rho_{c}$ has plummeted by some four orders of magnitude. Similarly, there is $\sim 10 \%$ change in $M_{\max }$ from the GRT to the NGT with $f_{\text {eff }}^{2}=5 \times 10^{-43} \mathrm{~cm}^{2}$, corresponding to a difference of an order of magnitude in $\rho_{c}$.

Just how fast the upper bound on $\rho_{c}$ varies with $f_{\text {eff }}^{2}$ is clear from Figure 3, where the parameter space for $\rho_{c}$ and $f_{\text {eff }}^{2}$ is displayed. All stable white dwarf solutions lie within the heavy solid line. For $f_{\text {eff }}^{2}<5 \times 10^{-44} \mathrm{~cm}^{2}$, the stability limit is identical to that in the GRT at $\rho_{c}=2.73 \times 10^{10} \mathrm{~g} \mathrm{~cm}^{-3}$. When $f_{\text {eff }}^{2}$ is large, the maximum $\rho_{c}$ goes like $\left(f_{\text {eff }}^{2}\right)^{-3 / 2}$. On the other hand, stars of a given mass tend to keep the same central density as in the GRT up to a $f_{\text {eff }}^{2}$ that brings them quite close to the onset of instability. We also find that $M_{\max }$ decreases with $f_{\text {eff }}^{2}$, and we can establish an upper bound of $5 \times 10^{-42} \mathrm{~cm}^{2}$ on $f_{\text {eff }}^{2}$, simply by requiring the existence of 1.0 $M_{\odot}$ dwarfs, two standard deviations away from the value of $1.053 \pm 0.028 M_{\odot}$ (Gatewood \& Gatewood 1978) for Sirius B

In Figure 3, the dashed line represents the solutions with vanishing gravitational redshift $\Delta \lambda / \lambda$. In the NGT,

$$
\begin{aligned}
\frac{\Delta \lambda}{\lambda} & =\left[g_{00}(R)\right]^{-1 / 2}-1 \\
& =\left[\left(1-\frac{2 G M}{c^{2} R}\right)\left(1+\frac{L^{4}}{R^{4}}\right)\right]^{-1 / 2}-1 \\
& \approx \frac{G M}{c^{2} R}-\frac{L^{4}}{2 R^{4}} .
\end{aligned}
$$

Between the dashed line and the stability boundary, solutions are such that $L^{4} / R^{4}>2 G M / c^{2} R$, and their light is gravitationally blueshifted.

A plot of $\Delta \lambda / \lambda$ against $\rho_{c}$ (Fig. 4) shows, however, that the blueshifted region is ruled out by observations. The NGT curves stray away from the GRT curve until they hit the stability limit (heavy line) or become negative, which can happen only for $\rho_{c}<3.5 \times 10^{7}$ $\mathrm{g} \mathrm{cm}^{-3}$. The two crosses represent data for two stars, Sirius B (Greenstein, Oke, \& Shipman 1971; Gatewood \& Gatewood 1978; Shipman 1979), and 40 Eridani B (Heintz 1974; Shipman 1979; Wegner 1980), whose mass and radius are fairly well known because they belong to binary systems. Fitting the Sirius B data, $\Delta \lambda / \lambda=(2.97 \pm 0.53) \times 10^{-4}$, to within the quoted error imposes $f_{\text {eff }}^{2}<2.3$ $\times 10^{-42} \mathrm{~cm}^{2}$. This must supersede the previous bound of $6 \times 10^{-42} \mathrm{~cm}^{2}$. As for 40 Eridani B, the GRT prediction lies two standard deviations below the measured value, $\Delta \lambda / \lambda=(7.97 \pm 0.43) \times 10^{-5}$. For the NGT to do as well requires that $f_{\text {eff }}^{2}<2 \times 10^{-42} \mathrm{~cm}^{2}$. All blueshifted solutions, which occur in the stable region only when $f_{\text {eff }}^{2}>5.33 \times 10^{-42} \mathrm{~cm}^{2}$, are therefore excluded.

Figures 2 and 3 show that the NGT decreases stability. The reason for this can be understood in terms of the NGT contribution to the force acting on a fluid element (see eq. [10c]), which we find to be attractive and, of course, proportional to $f_{\text {eff }}^{2}$. Larger pressures and densities are then required to support stars against the slightly increased gravitational force, thus accounting for the

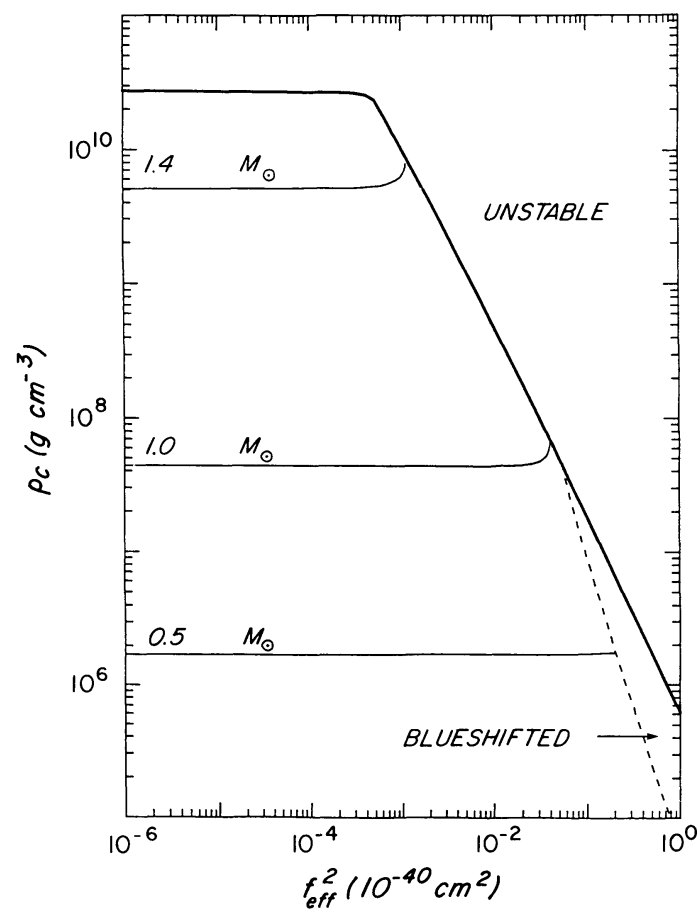

FIG. 3

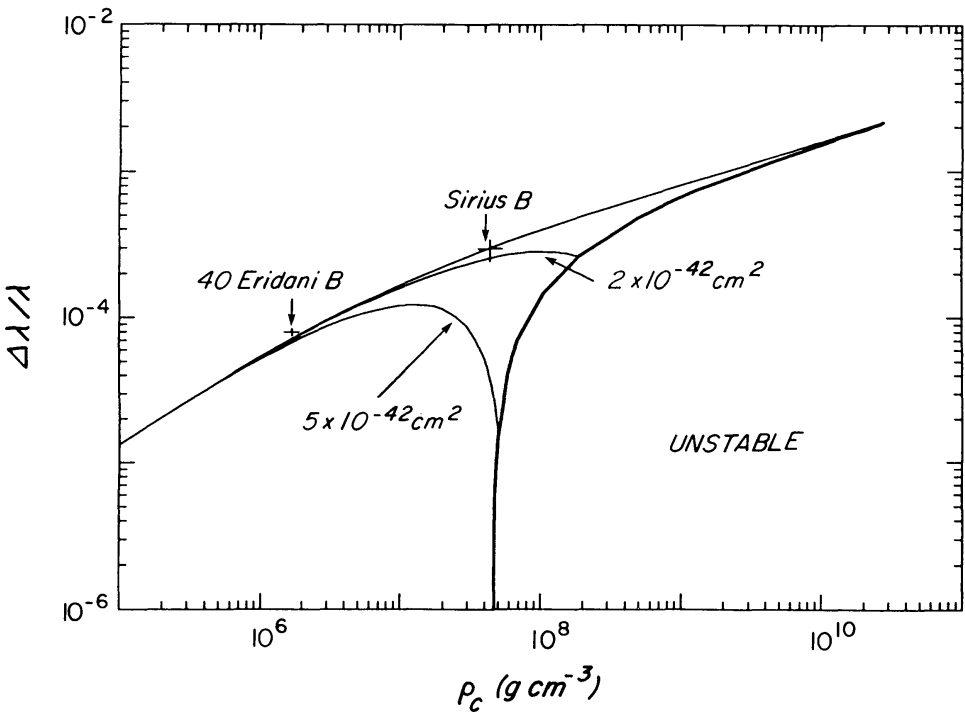

FIG. 4

Fig. 3. - The parameter space for white dwarfs in the NGT. The heavy solid line shows the maximum stable $\rho_{c}$ for each $f_{\text {eff }}^{2}$. The dashed line represents solutions with zero redshift. Also shown are solutions with constant mass $0.5,1$, and $1.4 M_{\odot}$.

Fig. 4. - The variation of the GRT and NGT gravitational redshift in white dwarfs, plotted against $\rho_{c}$. Two series of constant $f_{\text {eff }}^{2}$ solutions are included, and the heavy solid line marks the stability limit. The crosses represent data for Sirius B and 40 Eridani B. 


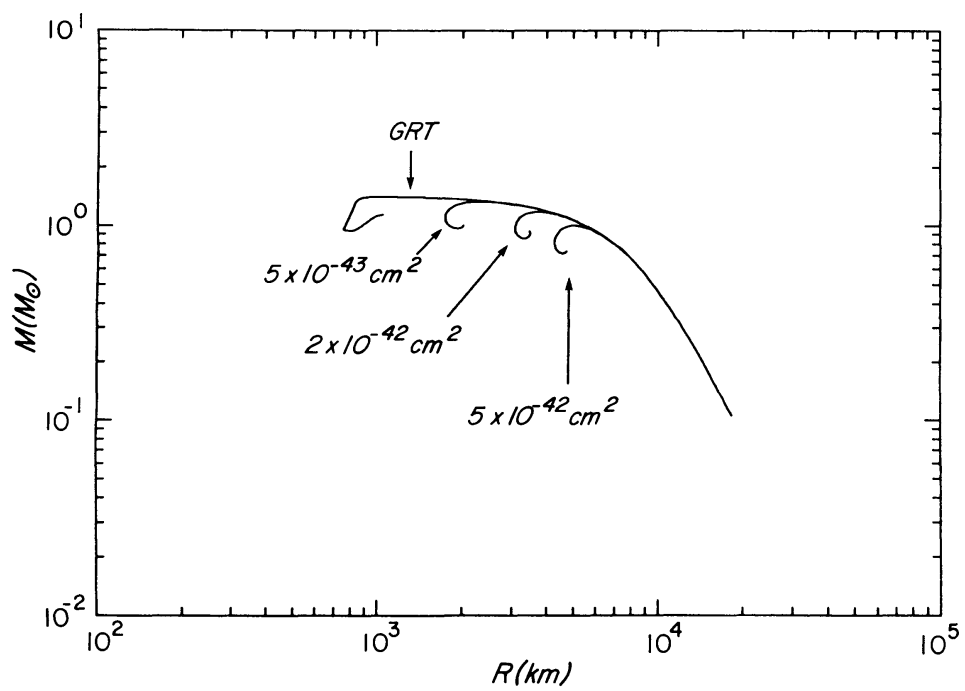

FIG. 5.-The mass-radius relation for white dwarfs in the GRT and in the NGT for the same three values of $f_{\text {eff }}^{2}$ as in Fig. 2

upturn of the lines of constant mass in Figure 3. Note that because $f_{\text {eff }}^{2}<2 \times 10^{-42} \mathrm{~cm}^{2}$, the central densities of NGT white dwarfs are quite close to those in the GRT for the same mass, so that their internal structure is not significantly altered by the NGT.

A larger attractive force should also lead to a smaller radius than in the GRT for a given mass. In fact, as the graph of $M$ versus $R$ in Figure 5 shows, a NGT white dwarf of a given mass cannot have its radius deviating very far from its GRT value before the onset of instability. The minimum radius $R_{\min }$ experiences a large increase, though: from $975 \mathrm{~km}$ in the GRT to $3000 \mathrm{~km}$ in the NGT when $f_{\text {eff }}^{2}=2 \times 10^{-42} \mathrm{~cm}^{2}$. This is made more explicit in a graph of $R$ versus $f_{\text {eff }}^{2}$ (Fig. 6), where we can see how quickly $R_{\text {min }}$ varies with $f_{\text {eff }}^{2}$ from the heavy line which again marks the boundary of the stable solution space.

As expected, the total NGT charge $L^{2}$ depends linearly on $f_{\text {eff }}^{2}$. The above bound on $f_{\text {eff }}^{2}$ and the stability criteria constrain $L^{2}$ so that $L_{\max }=530 \mathrm{~km}$ for any white dwarf, and $L_{\max }=490 \mathrm{~km}$ for dwarfs of $1 M_{\odot}$. If $f_{\text {eff }}^{2}$ is held fixed, $L^{2}$ varies linearly with the mass $M$ throughout the stable region.

Finally, in Figure 7, we have plotted the GRT and NGT density profiles for a $1 M_{\odot}$ white dwarf just before the onset of instability (for $f_{\text {eff }}^{2}=5 \times 10^{-42} \mathrm{~cm}^{2}$ ) in the NGT star, along with profiles of the quantities $s(r)$ and $F(r)\left[\equiv l^{4} /\left(l^{4}+r^{4}\right)\right]$ involved in equations (10). Notice how little the behavior of $\rho_{\mathrm{NGT}}$ and $\rho_{\mathrm{GRT}}$ differ. For $r<0.2 R, s$ and $F$ grow like $r^{2}$, as predicted by the analytical solution (12), valid for small $r$. Then they level out and begin to decrease. Near the edge, we expect that $F \approx L^{4} /\left(L^{4}+r^{4}\right)$, with $s$ quickly dropping to zero, as indeed the curves show.

\section{NEUTRON STAR SOLUTIONS}

As in the GRT, two types of stable high-density bodies exist in the NGT. Different as neutron stars may be from the white dwarfs studied in the previous section, the NGT affects their behavior-compared with the GRT-in much the same way. Again, a plot of

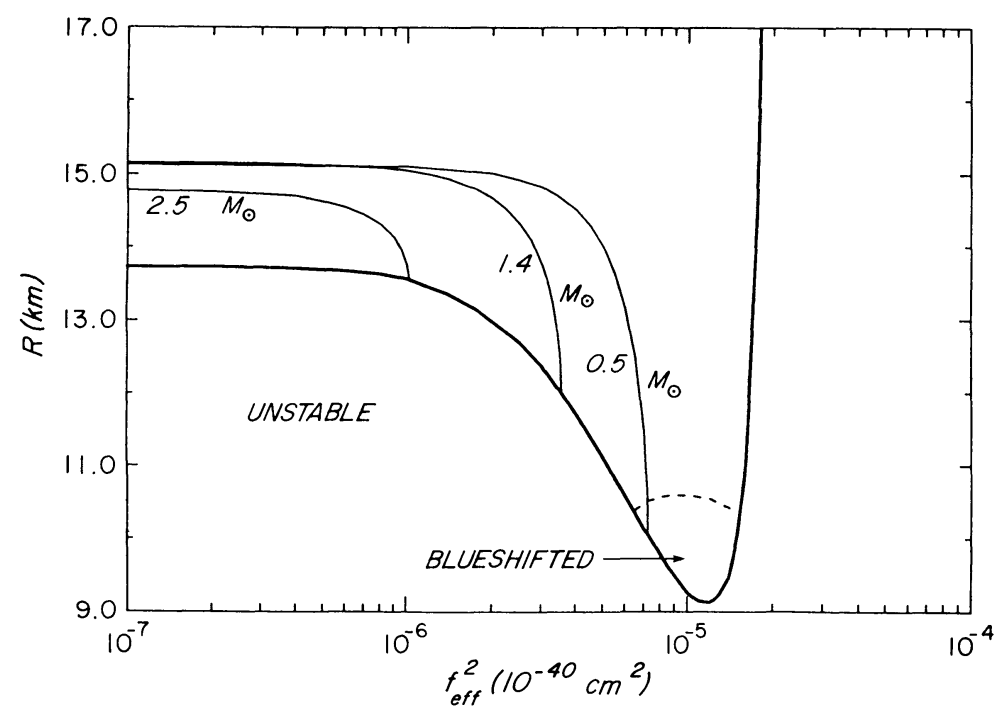

Fig. 6.-The variation of $R$ with $f_{\text {eff }}^{2}$ in NGT white dwarfs. The curves are as described in Fig. 3. 


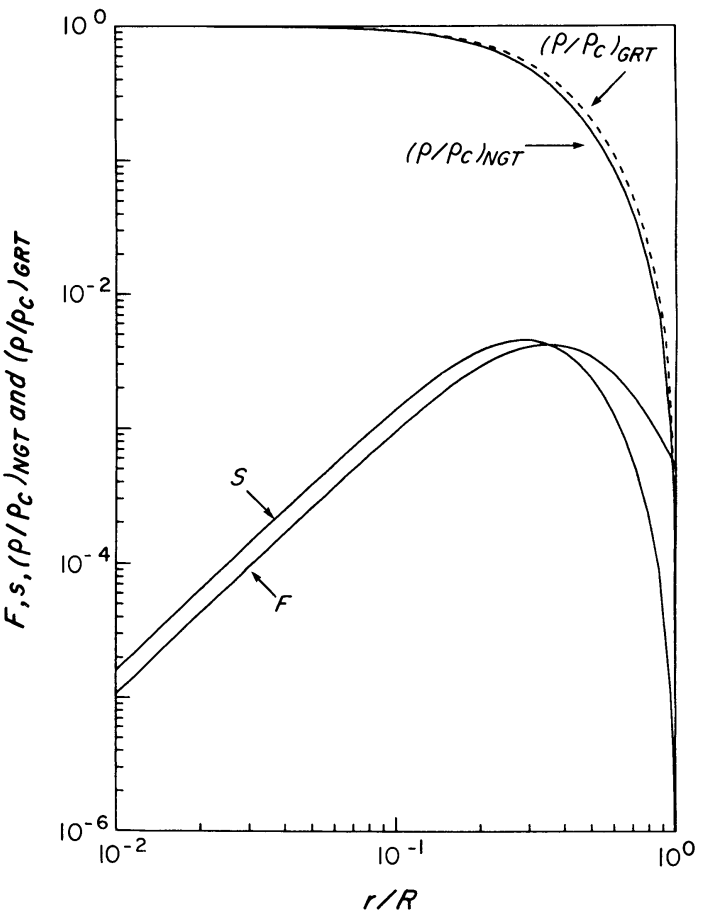

FIG. 7

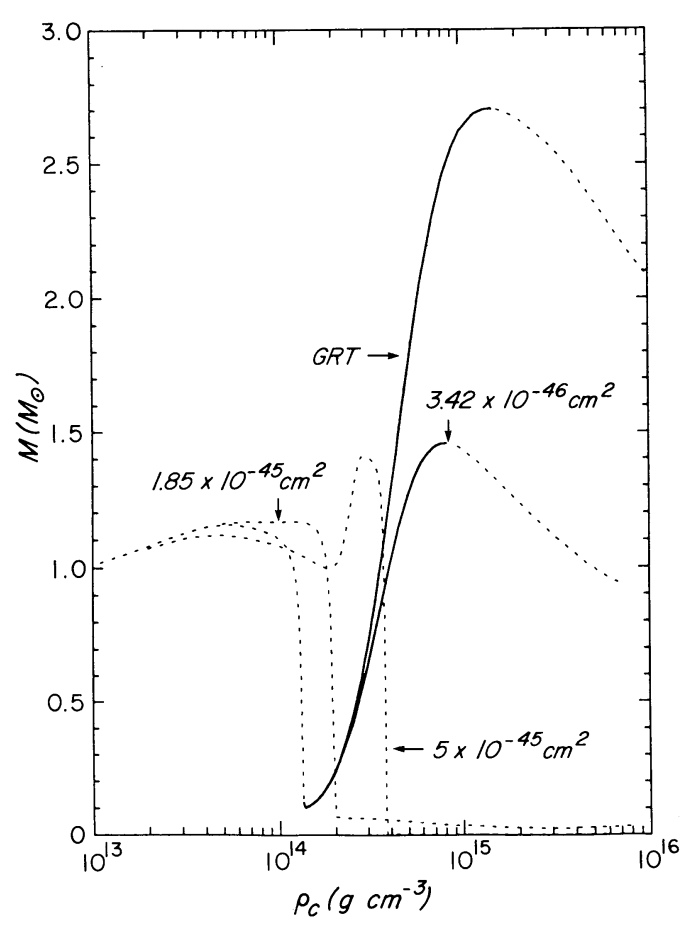

Fig. 8

FIG. 7.-Density profiles in GRT (dashed line) and NGT white dwarfs. Profiles for the NGT parameters $s$ and $F\left[\equiv l^{4} /\left(l^{4}+r^{4}\right)\right]$ introduced in $\S 2$ are also drawn.

FIG. 8. - The variation of mass with central density in GRT and NGT neutron stars. Stable solutions are represented by solid lines and unstable ones by dashed lines. Constant $f_{\text {eff }}^{2}$ curves are shown for $M_{\max }=1.45 M_{\odot}\left(3.42 \times 10^{-46} \mathrm{~cm}^{2}\right)$, for the absolute stability limit in any star $\left(1.85 \times 10^{-45} \mathrm{~cm}^{2}\right)$, and for a typical completely unstable set of solutions $\left(5 \times 10^{-45} \mathrm{~cm}^{2}\right)$.

mass $M$ against central density $\rho_{c}$ (Fig. 8) provides a good illustration of this. As $f_{\text {eff }}^{2}$ increases from its GRT value of zero, the neutron star peak gradually shrinks down, and it disappears when $f_{\text {eff }}^{2}=1.85 \times 10^{-45} \mathrm{~cm}^{2}$. There is no stable solution beyond that point, even though regions with $\partial M / \partial \rho_{c}>0$ still occur. If $f_{\text {eff }}^{2}=5 \times 10^{-45} \mathrm{~cm}^{2}$, for instance, the slope of the radius, $\partial R / \partial \rho_{c}$ is positive at the point where $\partial M / \partial \rho_{c}$ becomes positive again, as can be seen in Figure 9, which displays $R$ versus $\rho_{c}$ curves for the GRT and the same three values of $f_{\text {eff }}^{2}$ as in Figure 8. In both figures, only the solid lines correspond to stable objects.

Figure 10 shows the stable $\rho_{c}-f_{\text {eff }}^{2}$ parameter space as the area enclosed by the heavy line. The three constant mass lines make it clear that the NGT can have far more significant effects on neutron stars than on white dwarfs before the onset of instability. Here also the maximum value of $\rho_{c}$ decreases with $f_{\text {eff }}^{2}$, but not quite as fast. A small portion of the stable solution space is blueshifted, but this would concern only stars with masses of $0.5 \mathrm{M}_{\odot}$ or lower at their highest possible $f_{\text {eff }}^{2}$ and is therefore probably irrelevant. It is apparent that, as $f_{\text {eff }}^{2}$ increases, a larger central density is required to produce a body with the same mass. Equivalently, at constant $\rho_{c}$, an increase in $f_{\text {eff }}^{2}$ results in a decrease of the total mass. The maximum $f_{\text {eff }}^{2}$ that will allow observed neutron stars to exist is $3.42 \times 10^{-46} \mathrm{~cm}^{2}$, with a $M_{\max }$ of $1.45 M_{\odot}$, close to the mass of the primary component of PSR $1913+16$ (Weisberg $\&$ Taylor 1984). This particular solution has a central density of $8.15 \times 10^{14} \mathrm{~g} \mathrm{~cm}^{-3}$, instead of $4.42 \times 10^{14} \mathrm{~g} \mathrm{~cm}^{-3}$ in the GRT for a $1.45 M_{\odot}$ star. The solutions with $f_{\text {eff }}^{2}=3.42 \times 10^{-46} \mathrm{~cm}^{2}$ are also shown as one of the $M$ versus $\rho_{c}$ curves of Figure 8 .

From what precedes we are not surprised to discover that the radius of NGT neutron stars of a given mass can be substantially lower than in the GRT. Indeed, this is obvious from both Figure 11 ( $R$ vs. $\left.f_{\text {eff }}^{2}\right)$ and Figure $12(M$ vs. $R)$, where the stable solutions again lie within the heavy solid line. With $f_{\text {eff }}^{2}=3.42 \times 10^{-46} \mathrm{~cm}^{2}$, a $1.45 M_{\odot}$ body would see its radius reduced to $12.1 \mathrm{~km}$, some $20 \%$ smaller than in the GRT. In a $0.5 M_{\odot}$ star, for which a higher $f_{\text {eff }}^{2}\left(\approx 7 \times 10^{-46} \mathrm{~cm}^{2}\right)$ is allowed, the shrinkage could reach as much as thirty percent.

Figure 13 displays a graph of the NGT charge $L^{2}$ plotted against $M$ for stable neutron stars. The stability criteria rule out stars for which $L>8.1 \mathrm{~km}$. Interestingly enough, this turns out to be very close to the value of $L$ for $1.45 M_{\odot}$ objects when $f_{\text {eff }}^{2}=3.42$ $\times 10^{-46} \mathrm{~cm}^{2}$. Larger values of $f_{\text {eff }}^{2}$ merely decrease the mass and do not allow the NGT charge to build up as high. Equally noteworthy is the fact that for stable solutions, $L^{2}$ is still proportional to the mass, in spite of the strong nonlinearity of the field equations.

\section{DISCUSSION}

The main effect of the NGT on both white dwarfs and neutron stars is to lessen their stability compared to the GRT. At a given central density, both the mass and the radius decrease as a function of the coupling strength $f_{\text {eff }}^{2}$; equivalently, a higher central density is needed to support a star of a given mass against collapse. One can just as well say that the NGT acts as if a softer equation of state were being used. The explanation of this behavior is that the net NGT contribution to the force exerted on a fluid element in a body of uniform composition is attractive. 


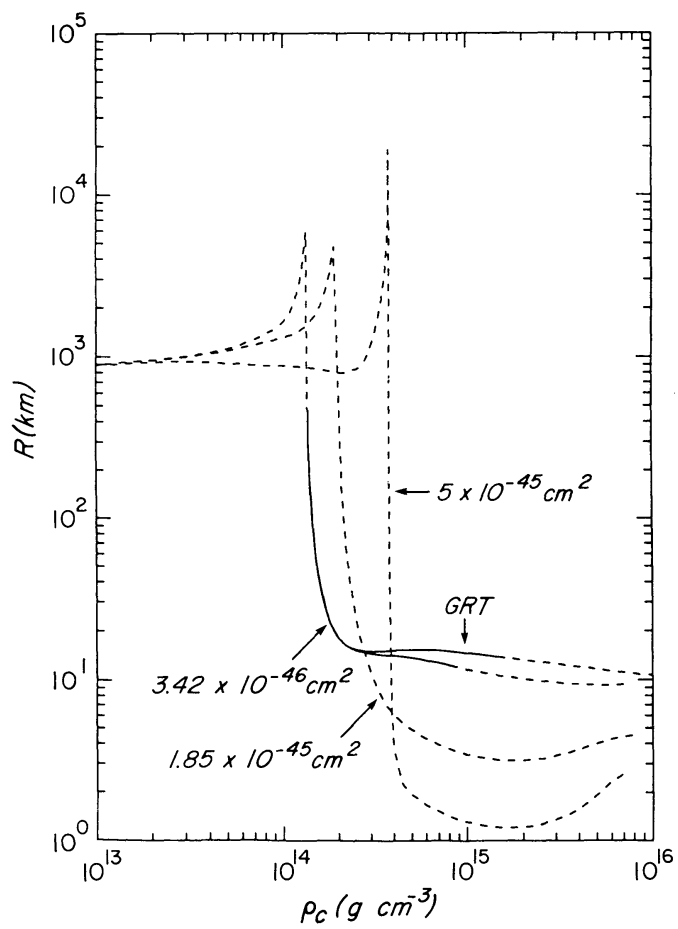

Fig. 9

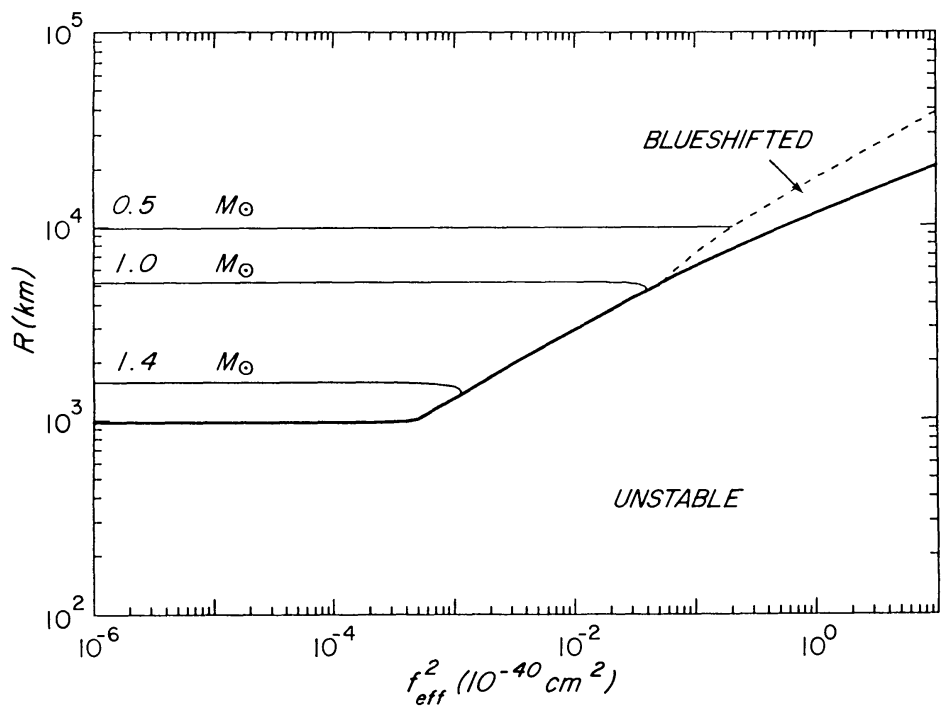

FIG. 10

FIG. 9.-The variation of the neutron star radius plotted against $\rho_{c}$ in the GRT and the NGT. Curves are as described in Fig. 8 .

FIG. 10.-The parameter space for neutron stars in the NGT. The heavy solid line shows the maximum and minimum stable $\rho_{c}$ for each $f_{\text {eff }}^{2}$. The dashed line represents solutions with zero redshift. Also shown are solutions with constant mass $0.5,1.4$, and $2.5 M_{\odot}$.

Bounds can also be set on $f_{\text {eff }}^{2}$. Assuming that all white dwarfs have the same composition, from the redshift data we can put $f_{\text {eff }}^{2}<2 \times 10^{-42} \mathrm{~cm}^{2}$, which allows objects as massive as $1.18 M_{\odot}$ to exist. If white dwarfs with larger masses are ever observed, this will put a more stringent limit on their $f_{\text {eff }}^{2}$. It has been suggested (Moffat 1990a) that the companion in the binary system PSR $1913+16$ is actually a white dwarf with a mass of $1.387 M_{\odot}$. If this were true, the maximum $f_{\text {eff }}^{2}$ for white dwarfs would go down to $1.77 \times 10^{-43} \mathrm{~cm}^{2}$.

In neutron stars there is a maximum value for $f_{\text {eff }}^{2}, 1.85 \times 10^{-45} \mathrm{~cm}^{2}$, above which no stable star can exist. Moreover, in objects of $1.45 M_{\odot}, f_{\text {eff }}^{2}<3.42 \times 10^{-46} \mathrm{~cm}^{2}$. Because neutron stars of different mass have different mixtures of protons, neutrons, and electrons, one cannot immediately claim that this limit holds for all neutron stars. Indeed, equation (8) can be written

$$
f_{\text {eff }}^{2}=\left[f_{n}^{2}+y\left(f_{p}^{2}+f_{e}^{2}\right)\right] /(1+y m),
$$

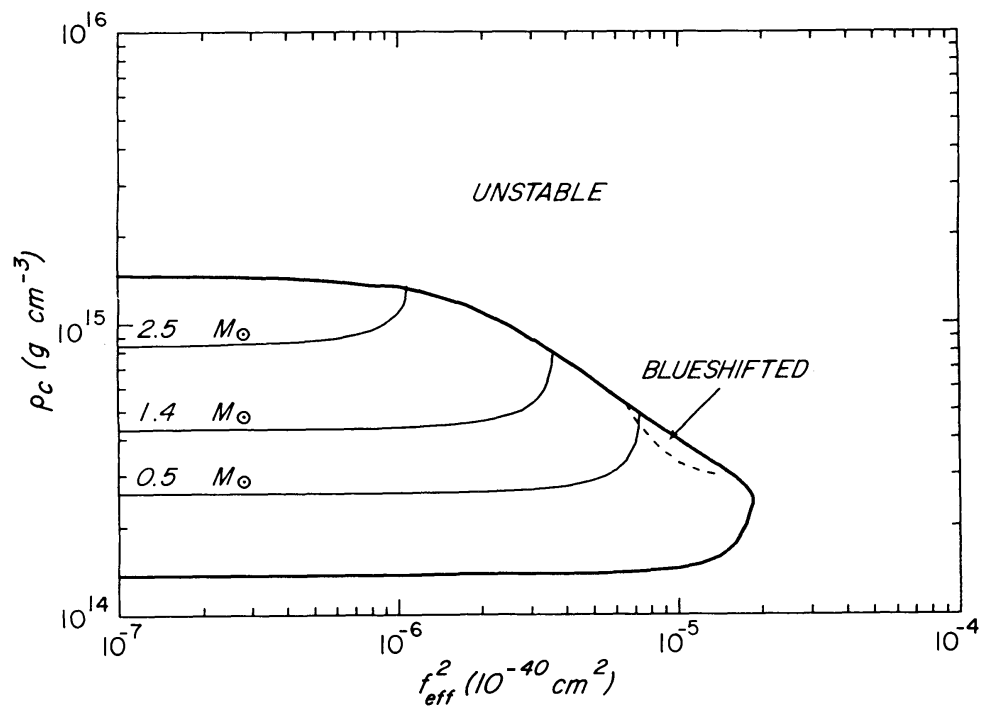

FIG. 11.-The variation of $R$ with $f_{\text {eff }}^{2}$ in NGT neutron stars. The curves are as described in Fig. 10. 


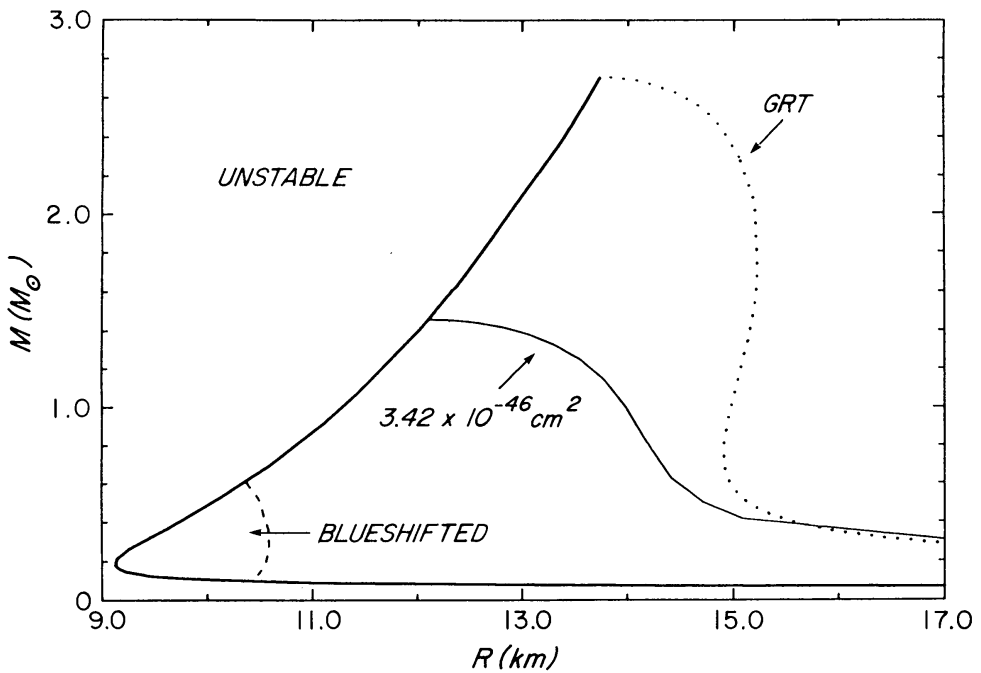

FIG. 12. - The mass-radius relation for neutron stars in the GRT and in the NGT for $f_{\text {eff }}^{2}=3.42 \times 10^{-46} \mathrm{~cm}^{2}$. The heavy solid line and the dashed line are as in Fig. 10.

where $y$ stands for the ratio of proton to neutron densities, $n_{p} / n_{n}$, and $m \equiv\left(m_{p}+m_{e}\right) / m_{n}$. In white dwarfs, $y=1$ and, noting that $f_{\text {eff }}^{2}$ need not be positive,

$$
\left|\left(f_{n}^{2}+f_{p}^{2}+f_{e}^{2}\right) /(1+m)\right|<2 \times 10^{-42} \mathrm{~cm}^{2} .
$$

In order to produce a $1.45 \mathrm{M}_{\odot}$ neutron star, $f_{\text {eff }}^{2}$ for that star cannot be higher than $3.42 \times 10^{-46} \mathrm{~cm}^{2}$. Such a star has a central density of $8.15 \times 10^{14} \mathrm{~g} \mathrm{~cm}^{-3}$. Hence,

$$
\left|\left[f_{n}^{2}+y_{\mathrm{NS}}\left(f_{p}^{2}+f_{e}^{2}\right)\right] /\left(1+y_{\mathrm{NS}} m\right)\right|<3.42 \times 10^{-46} \mathrm{~cm}^{2} .
$$

Combining this with equation (15) and setting $m$ equal to 1 yields

$$
\begin{aligned}
\left|f_{p}^{2}+f_{e}^{2}\right| & <\left[2 f_{\mathrm{WD}}^{2}-\left(1+y_{\mathrm{NS}}\right) f_{\mathrm{NS}}^{2}\right] /(1-y) \\
\left|f_{n}^{2}\right| & <\left[2 y f_{\mathrm{WD}}^{2}-\left(1+y_{\mathrm{NS}}\right) f_{\mathrm{NS}}^{2}\right] /(1-y),
\end{aligned}
$$

in which $f_{\mathrm{WD}}^{2}=2 \times 10^{-42} \mathrm{~cm}^{2}$, and $f_{\mathrm{NS}}^{2}=3.42 \times 10^{-46} \mathrm{~cm}^{2}$.

For neutron stars with free neutrons, protons, and electrons in chemical equilibrium, $y_{\mathrm{NS}}$ varies from 0.004 to 0.02 over the density range $(0.2-1.5) \times 10^{15} \mathrm{~g} \mathrm{~cm}^{-3}$ in which stable stars exist. In the solution we are using, $y_{\mathrm{Ns}} \approx 0.012$ over most of the star. Thus, to a

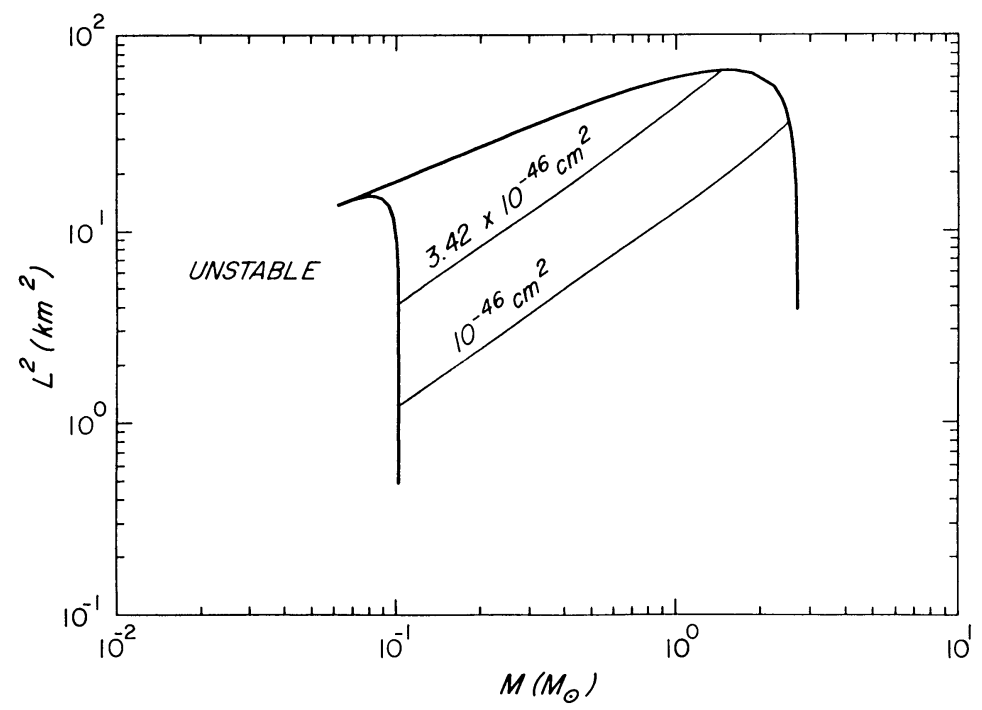

FIG. 13.-The $L^{2}-M$ relation for NGT neutron stars. The stability limit is drawn as a heavy solid line. Two sets of solutions with constant $f_{\text {eff }}^{2}$ are also shown. 
good approximation,

$$
\begin{aligned}
\left|f_{p}^{2}+f_{e}^{2}\right| & <2 f_{\mathrm{WD}}^{2} \\
& <4 \times 10^{-42} \mathrm{~cm}^{2}, \\
\left|f_{n}^{2}\right| & <2 y_{\mathrm{NS}} f_{\mathrm{WD}}^{2} \\
& <5 \times 10^{-44} \mathrm{~cm}^{2} .
\end{aligned}
$$

As long as $f_{\mathrm{WD}}^{2} \gg f_{\mathrm{NS}}^{2}$, the upper bound on $f_{n}^{2}$ is insensitive to $f_{\mathrm{NS}}^{2}$. It is also quite obvious that the limit on $f_{\mathrm{eff}}^{2}$ for a $1.45 M_{\odot}$ neutron star does not prevent $f_{\text {eff }}^{2}$ from reaching larger values for lower mass stars. Taking $f_{\text {eff }}^{2} \approx f_{n}^{2}+y_{\text {NS }}\left(f_{p}^{2}+f_{e}^{2}\right)$, we can imagine a situation with $f_{p}^{2}+f_{e}^{2} \approx-3 \times 10^{-44} \mathrm{~cm}^{2}$ and $f_{n}^{2} \approx 7 \times 10^{-46} \mathrm{~cm}^{2}$ which would produce a sufficiently low $f_{\text {eff }}^{2}$ at the density required for a $1.45 M_{\odot}$ star while allowing larger values of $f_{\text {eff }}^{2}$ at lower densities. Therefore, the most reliable limit for the $f_{\text {eff }}^{2}$ of a neutron star of a given mass is determined essentially by the stability criteria.

In most neutron stars, such as the primary component of PSR $1913+16$, rotational effects that help support them and increase their allowable mass are expected to be too small (Hartle 1973) to change $f_{\mathrm{NS}}^{2}$ appreciably, and certainly not large enough to invalidate equation (18). In white dwarfs, on the other hand, such effects could increase $f_{\text {wD }}^{2}$ by a significant amount, but only if Sirius B or 40 Eridani B rotated rapidly enough, which does not appear to be the case. If a new $f_{\text {wD }}^{2}$ were ever inferred from a fastly rotating white dwarf, those corrections would have to be taken into account. Incidentally, the NGT lets neutron stars rotate faster without breaking up (Moffat 1990b); one is not forced to soften the equation of state so much in order to compensate for the centrifugal force.

As a consequence of our bounds on $f^{2}$, and to the extent that equation (6) provides a valid description of $S^{\mu}$, we are able to rule out ordinary matter as a source of NGT force sufficiently strong to produce periastron shifts significantly different from those predicted in the GRT for main-sequence binary systems. Indeed, assume a coupling strength $\left|f_{p}^{2}+f_{e}^{2}\right|$ as high as $4 \times 10^{-42} \mathrm{~cm}^{2}$. The total NGT charge of a main-sequence star composed only of protons, electrons, and neutrons, is

$$
L^{2}=N\left[\left(f_{p}^{2}+f_{n}^{2}\right) Z / N+f_{n}^{2}\right],
$$

where $Z$ refers to the number of protons and $N$ the number of neutrons. Now $N$ is related to the mass of the star by $M=m_{p} N(Z / N$ +1 ), where $Z / N \approx 7.7$ for the DI Her and AS Cam systems ( $m_{p}$ is the proton mass). Thus, in a $5 M_{\odot}$ star, $\left|L^{2}\right|$ is at most $9 \times 10^{16}$ $\mathrm{cm}^{2}$, leading to $L \approx 3000 \mathrm{~km}$ for each of the components of DI Her; this is much too small (Moffat 1987, 1989) to account for the reported periastron shift observations of Guinan \& Maloney (1985). A model using cosmions as the effective NGT source has been shown by Moffat (1989) to fit the data, however.

If a softer equation of state were used instead of the mean-field variant chosen here, the limits could only go down, not up. Since our EOS is just about the stiffest one, we can claim that they are the highest ones possible. Moreover, the fact that, according to the NGT, a $1.45 M_{\odot}$ neutron star with a core density as high as $8.1 \times 10^{14} \mathrm{~g} \mathrm{~cm}^{-3}$ can exist, has interesting implications. The magnitude of the binding energy of such an object is some $25 \%$ larger than the Newtonian potential energy. This is sure to have a strong effect on the dynamics of supernova explosions, since the implosion phase creating that core would store appreciably more energy to drive the shock wave of the subsequent explosion (see also Moffat 1991).

This work was supported by the Natural Sciences and Engineering Research Council of Canada. One of us (P. S.) wishes to thank the Instituut voor Theoretische Fysika of the Katholieke Universiteit in Leuven, Belgium, where part of this work was carried out, for their kind hospitality.

\section{REFERENCES}

Baym, G., Bethe, H. A., \& Pethick, C. J. 1971a, Nucl. Phys. A, 175, 225

Baym, G., Pethick, C., \& Sutherland, P. 1971b, ApJ, 170, 299

Campbell, L. 1988, Ph.D. thesis, University of Toronto

Chandrasekhar, S. 1939, An Introduction to Stellar Structure (Chicago: University of Chicago Press)

Damour, T., \& Taylor, J. H. 1991, ApJ, 366, 501

Gatewood, G. D., \& Gatewood, C. V. 1978, ApJ, 225, 191

Greenstein, J. L., Oke, J. B., \& Shipman, H. L. 1971, ApJ, 169, 563

Guinan, E. F., Carroll, S. M., Lallo, M. D., Baliunas, S. L., Loeser, J. G., \& Donahue, R. A. 1987, preprint, Villanova University

Guinan, E. F., \& Maloney, F. P. 1985, AJ, 90, 1519

. 1989, AJ, 98, 1800

Harrison, B. K., Thorne, K. S., Wakano, M., and Wheeler, J. A. 1965, in Gravitation Theory and Gravitational Collapse (Chicago: University of Chicago Press), p. 50

Hartle, J. B. 1973, Ap\&SS, 24, 385

Heintz, W. D. 1974, AJ, 79, 819

Hindmarsh, A. C. 1980, ACM-SIGNUM Newsletter, 15, No. 4

Krisher, T. P. 1985, Phys. Rev. D, 32, 329
Maloney, F. P., Guinan, E. F., and Boyd, P. T. 1989, AJ, 98, 1800

Moffat, J. W. 1979, Phys. Rev. D, 19, 3554 1987, Phys. Rev. D, 35, 3733

1989, Phys. Rev. D, 39, 474

1990a, in Proc. of the XXV Rencontre de Moriond, Les Arcs, France, ed. O. Fackler and J. Tran Thanh Van (Gif sur Yvette: Editions Frontières), in press

1990b, ApJ, 347, L59

1991, in Gravitation 1990: A Banff Summer Institute, ed. R. B. Mann \& P. Wessa (Singapore: World Scientific), in press Moffat, J. W., \& Woolgar, E. 1988, Phys. Rev. D, 37, 918

Pandharipande, V. R., Pines, D., \& Smith, R. A. 1976, ApJ, 208, 550

Savaria, P. 1989, Classical Quantum Grav., 6, 1003

Savaria, P. 1989, Classical Quan

Shipman, H. L. 1979, ApJ, 228, 240

Taylor, J. H., \& Weisberg, J. M. 1989, ApJ, 345, 434

Vincent, D. 1985, Classical Quantum Grav., 2, 409

Wegner, G. 1980, AJ, 85, 1255

Weisberg, J. M., \& Taylor, J. H. 1984, Phys. Rev. Letters, 52, 1348 